\title{
Efecto de la ingesta sobre la volemia durante la sesión de hemodiálisis
}

\author{
Patricia Molina Mejías, María Trinidad Quesada Armenteros, Sonia Ruiz Almería, Beatriz Liébana Pamos, \\ Miriam Esquinas Marta, M $^{\mathrm{a}}$ Amor Martínez Aranda
}

Servicio de Nefrología, Hospital Universitario Infanta Leonor. Madrid. España

\section{Resumen}

Introducción: Desde el punto de vista enfermero nos planteamos si los pacientes pueden o no ingerir alimentos durante la sesión de hemodiálisis.

Objetivo: Con este estudio nos proponemos evaluar la repercusión hemodinámica de la ingesta intrahemodiálisis en relación con la caída de volumen sanguíneo y la tensión arterial.

Material y Método: Se realiza un estudio prospectivo, observacional, transversal en 22 pacientes adultos en programa de hemodiálisis crónica, recogiendo datos durante tres sesiones consecutivas valorando la repercusión hemodinámica en función de la ingesta alimenticia.

Resultados: La caída de volumen sanguíneo máxima media fue de $12 \%$ y la caída de volumen sanguíneo relacionada con la ingesta fue de $3.5 \%$.

No encontramos relación de mayor caída de volumen sanguíneo o tensión arterial con el tipo de ingesta durante la sesión, ni la cantidad ingerida.

Ningún paciente presentó episodio de hipotensión relacionado con la ingesta por lo que no encontramos diferencias estadísticamente significativas.

Conclusión: Concluimos el estudio observando que la ingesta durante la sesión de hemodiálisis produce una caída de volumen sanguíneo en la mayoría de los pacientes pero sin repercusión hemodinámica.

Correspondencia:

Patricia Molina Mejías

C/ Zazuar n 9, $3^{\circ} \mathrm{C} .28031$ Madrid. España

E-mail: ey_frey_7@hotmail.com
PALABRAS CLAVE

- VOLEMIA

- INGESTA

- HEMODIÁLISIS

Effect of intake on blood volume during hemodialysis session

\section{Abstract}

Introduction: Nurse's angle, we appear if the patients can consume food or not during the hemodialysis sessions.

Objective: With this study we propose to evaluate the haemodynamic effect of overall intake in hemodialysis sessions in relation with the blood volume and blood pressure decreased.

Material and method: This was a prospective, observational, cross-sectional study. There were 22 chronic hemodialysis patients. We collected information during three consecutive dialysis sessions valuing the haemodynamic effect depending on overall intake.

Results: The maximum average fall of blood volume was $12 \%$ and the fall of blood volume related to the overall intake was $3.5 \%$. We didn't find relation of major fall of blood volume or blood pressure with the type of overall intake during the session, nor the ingested quantity. No patient presented episode of hypotension related to overall intake, so we didn't find statistically significant differences.

Conclusion: We conclude the study observing that overall intake during the haemodialysis produces a fall of blood volume in the majority of the patients but without haemodynamic effect. 


\section{KEYWORDS \\ - VOLUME \\ - OVERALL INTAKE \\ - HEMODIALYSIS}

\section{Introducción}

Entre las preocupaciones de la enfermería durante la sesión de hemodiálisis (HD) figura el asegurar una buena tolerancia hemodinámica. Unos de los riesgos más habituales durante la sesión es el descenso de presión arterial (PA). Esta caída de PA causa en los pacientes disconfort, reduce la eficacia del tratamiento y aumenta la morbilidad. La hipotensión repetida sintomática puede producir daños cerebrales y cardiacos entre otros ${ }^{1}$.

Para evitar estas hipotensiones intradiálisis y gracias disponemos de un biosensor de la caída de volumen sanguíneo BVM (Blood Volume Monitor), una herramienta automática y no invasiva para monitorizar el volumen sanguíneo. Durante el tratamiento la ultrafiltración reduce el agua plasmática aumentando el hematocrito, que es detectado por el módulo BVM y detectado como una caída relativa del volumen sanguíneo (figura 1). Esta caída precede a la hipotensión, permitiendo una intervención preventiva² ${ }^{2}$. a los avances tecnológicos en las máquinas de HD

La hipotensión postprandial es definida como una caída de la presión arterial sistólica de $\geq 20 \mathrm{~mm} \mathrm{Hg} 0$ una reducción en la presión sistólica de $\leq 90 \mathrm{mmHg}$ cuando la prepandial es $>100 \mathrm{mmHg}$, en un plazo de dos horas desde la comida.

El inicio de la caída es evidente, por lo general casi de inmediato, pero puede ocurrir entre 15-75 minutos después de una comida ${ }^{5}$.

Sin embargo, pese a la importancia que parece tener, se han realizado muy pocos estudios sobre la influencia específica de la ingesta de la comida y bebida en la sesión de $\mathrm{HD}^{6}$.

Aunque en algunas unidades se prohíbe la ingesta debido a la posible hipotensión y riesgo de mala tolerancia, esta medida es poco aceptada en general entre los pacientes, por eso nos planteamos un estudio cuyo objetivo es evaluar la repercusión hemodinámica de la ingesta intradiálisis relacionando la caída de volumen sanguíneo (CVS) y la tensión arterial.

\section{Material y Método}

Nuestro estudio es un estudio prospectivo, observacional, transversal en pacientes en programa de hemodiálisis crónica realizado en un único centro de diálisis del Hospital Universitario Infanta Leonor entre los meses de noviembre y diciembre de 2015 en el que se incluyeron 22 pacientes. Se registraron un total de 66 sesiones, 3 sesiones consecutivas en cada paciente, la primera de ellas coincidiendo con la analítica mensual.

A todos los pacientes se les explicó, asegurándose de que entendían en que consistía el estudio, y se les dio la posibilidad de hacer preguntas al respecto o aclarar en caso de dudas. Todos firmaron el consentimiento informado.

Figura 1. Aumento en la concentración de elementos formes sanguíneos por ultrafiltración.

Uno de los factores que se asocia con la caída de PA durante las sesiones es la ingesta oral. En pacientes con fallo autonómico la ingesta puede causar hipotensiones sintomáticas, ello es debido a que la ingesta oral puede causar una vasodilatación asplácnica, esta hipotensión se conoce como hipotensión postpandrial ${ }^{3,4}$.
Los únicos criterios de inclusión fueron que los pacientes fueran mayores de 18 años y llevaran con tratamiento con hemodiálisis durante al menos 3 meses. Y como criterios de exclusión fueron la presencia de inestabilidad hemodinámica con hipotensiones frecuentes, contraindicación o problemas para la ingesta de alimentos.

Todos los pacientes se dializaron con el monitor de diálisis ST5008 (Fresenius ${ }^{\circledR}$, FMC, Bad Homburg, Ger- 
many) con el modulo de caída de volumen sanguíneo o BVM (Blood Volume Monitor ${ }^{\circledR}$ ), que permitió monitorizar la CVS durante la sesión de HD.

\section{Parámetros demográficos y de diálisis recogidos:} Se recogieron una serie de parámetros demográficos: edad, enfermedad de base, tiempo en diálisis, peso, técnica de diálisis: Hemodiálisis (HD) o Hemodialfiltración (HDF), frecuencia de la técnica, el tipo de acceso vascular: fístula (FAV) o catéter (CT), y la función renal residual (FRR) medida como el aclaramiento medio de urea y creatinina $((\mathrm{CCr}+\mathrm{CU}) / 2)$ en orina de 24 horas (Si este era $<1 \mathrm{ml} / \mathrm{min}$ o la diuresis $<100 \mathrm{ml} /$ día se consideró ausencia de FRR).

Los parámetros de diálisis: tiempo, flujo sanguíneo $(Q b)$, flujo del líquido de diálisis (Qd), conductividades de sodio y bicarbonato, temperatura del líquido, tipo de concentrado ácido, concentración de $\mathrm{K}$ y calcio en el LD, dializador, tipo y dosis de heparina, volúmenes de infusión en HDF, el Kt medido automáticamente por el biosensor $\mathrm{OCM}^{\circledR}$, la ultrafiltración (UF) por sesión, tasa de ultrafiltración, la ganancia interdiálisis (GID), las tensión arterial (TA) pre, horarias y post HD, FC. También se recogieron el número de hipotensiones definidas como toda disminución aguda de la presión arterial percibida por el paciente que precisara la intervención del personal de enfermería.

Se recogieron los alimentos ingeridos durante la sesión de diálisis: un vaso de leche o café con leche o infusión, una barrita de pan con mantequilla o galletas, yogur y pieza de fruta.

\section{Material y Método}

En el momento que el paciente comenzó la ingesta se procedió a activar el botón ingesta del monitor, no se realizó modificación alguna de la pauta habitual. Se anotó toda la alimentación ingerida durante la sesión de HD (volumen y calorías consumidas durante la sesión).

Se tomó la TA antes de iniciar la ingesta y a los $30 \mathrm{mi}-$ nutos de la misma. El resto de las constantes se tomaron de forma habitual

Al finalizar la sesión se analizó el porcentaje de CVS producido con la ingesta. Se consideró que el paciente tiene CVS en relación con la ingesta si la pendiente de la curva mostrada en el monitor se modifica + de un 1\% en relación con la ingesta.

\section{Tratamiento estadístico}

EI análisis estadístico se realizó con el programa SPSS 15.0. Las variables cualitativas se muestran con porcentajes y las variables cuantitativas como medias (desviación estándar) o medianas (mínimo-máximo). Los estudios de $t$ de student y Anova fueron utilizados para comparar variables cuantitativas.

Los test chi-cuadrado fueron utilizados para las variables cualitativas. Un valor de $p$ menor de 0,05 fue considerado como estadísticamente significativo.

\section{Resultados}

El total de la muestra del estudio fueron 22 pacientes. La edad era de 70.9 (12.2) [40-48] años. El $54.5 \%$ eran mujeres.

La principal etiología de la enfermedad renal crónica fue la nefropatía diabética 5 (22.7\%).

La técnica de hemodiálisis más utilizada fue HDF 19 (86.3\% [HDF postdilucional 18 (81.8\%), HDF mixta $1(4.5 \%)]$.

EI acceso vascular de los pacientes era: FAV 15 $(68.2 \%)$ y CT 7 (31.8\%).

La función renal residual (FRR) estaba presente en 6 pacientes $(27.3 \%)$.

El tiempo programado de diálisis fue 259 (10.5) [255-300] minutos.

La media de caída del volumen sanguíneo fue de 3.5 (2.1) [0-9.5] \%.

La CVS se mantuvo igual durante las 3 sesiones de hemodiálisis consecutivas. Solo a 3 pacientes de los 22 no les cayó el volumen sanguíneo en relación con la ingesta.

Ningún paciente presentó disminución de la TA a los 30 minutos de la ingesta con respecto a la TA preingesta sin presentar CVS. Así como tampoco presentaron hipotensión durante la sesión de HD según el criterio propuesto por la Guía K-DOQI.

No encontramos diferencias estadísticamente significativas en la CVS relacionada con la ingesta y la tasa de UF, la disminución de la TAS ni TAD, volumen in- 
gerido, tipo de alimento ingerido, Índice de Charlson, edad, ni UF ajustada al peso.

Si que encontramos una relación estadísticamente significativa con:

\section{- La FRR ( $p=0.048)$}

- El acceso vascular, con una mayor CVS en los pacientes que tiene $C T$ con respecto a los que tenían FAV $(p=0.001)$. Los 7 pacientes que tenían catéter presentaron una caída $\geq 2.5 \%$ en relación con la ingesta.

- El sexo quedó en el límite de la significación (0.65) con una mayor CVS en las mujeres (tabla 1).

\section{Discusión}

La ingesta oral por sí mismo no parece ser un factor suficiente para causar hipotensión, sin embargo, aumenta el número de eventos en aquellos pacientes que tienden a la hipotensión, especialmente cuando la ganancia de peso es elevada. Vázquez M. y cols $^{6}$ en su estudio prospectivo de 26 pacientes con ayuno controlado, en el que los pacientes eran controles de sí mismos, no encontraron asociación entre la ingesta y la aparición de hipotensión, si bien excluyeron a los pacientes inestables hemodinámicamente.

Benaroia $\mathrm{M}$ y cols ${ }^{3}$ en su estudio transversal de 126 pacientes no encontraron tampoco relación entre la ingesta oral y la hipotensión, si bien al analizar aquellos pacientes con ingesta superior a 0.2 L o mayor de 200 calorías encontró que la incidencia de hipotensión durante la diálisis era mayor.

En nuestro estudio encontramos que existe relación entre la caída de volumen sanguíneo, si bien no se produjeron hipotensiones. Ninguno de nuestros pacientes presentó disminución de tensión arterial pasados $30 \mathrm{mi}-$ nutos tras la ingesta, ni durante la sesión de hemodiálisis según el criterio propuesto por la guía K- DOQI, hemos de destacar que la incidencia de hipotensiones en nuestra unidad es muy baja, muy inferior a la descrita por otros autores.

Analizamos también la relación entre la CVS y el tipo de alimento, la cantidad de comida ingerida, la edad, el sexo, la ultrafiltración ajustada al peso y el índice de Charlson, sin encontrar correlaciones significativas.

Queremos destacar en nuestro trabajo la relación estadísticamente significativa encontrada entre la caída de volumen sanguíneo y la función renal residual. La menor caída de volumen en los que tienen función renal residual podría atribuirse a la menor ganancia de peso interdiálisis, que conllevaría una menor tasa de ultrafiltración programada y por tanto una menor hemoconcentración.

También, hallamos relación entre la caída de volumen sanguíneo y el acceso vascular. Los pacientes portadores de catéter

Abreviaturas: ERC: Enfermedad renal crónica; HD: hemodiálisis; HDF: hemodiafiltración; FAV: fístula arteriovenosa; CT: catéter tunelizado; CVS: Caída volumen sanguíneo; TAS: tensión arterial sistólica; TAD: tensión arterial diastólica.

Resultados presentados como media (Desviación estándar) [rango intercuartílico]. 
presentan una mayor caída de volumen sanguíneo, cuando se comparan con los portadores de FAV. Este resultado puede atribuirse a que su situación vascular es peor que la de los pacientes portadores de FAV.

En nuestra unidad permitimos la ingesta oral en nuestros pacientes, y este estudio apoya esta política, no obstante, hemos de considerar que excluimos del estudio a aquellos pacientes que presentaban inestabilidad hemodinámica con hipotensiones frecuentes, 0 contraindicación o problemas para la ingesta de alimentos. En el estudio de Zoccali $\mathrm{C}^{4}$ centrado en el hábito de comer durante la diálisis, en una encuesta realizada en 12 centros renales de Italia, solo 2 de estos, prohíben la ingesta durante la sesión de hemodiálisis, y el $70 \%$ de los pacientes del resto de los centros consumía un snack durante la sesión.

Nuestro estudio tiene no obstante algunas limitaciones como el que solo hemos recogido datos de tres sesiones, aunque el número de pacientes incluidos es bastante representativo, si bien tampoco hemos tenido en cuenta si ha habido variación de peso seco en esas sesiones. Tampoco hemos controlado a los pacientes con ayuno en diálisis por lo impopular de la medida, y a aquellos pacientes que no ingerían por que no es ético si tienen contraindicación medica para la ingesta oral.

\section{Agradecimientos}

Queremos agradecer ante todo el esfuerzo realizado por todos nuestros compañeros de la Unidad de Hemodiálisis del Hospital Infanta Leonor quienes nos ayudaron a recoger todos los datos de nuestro estudio, a nuestra supervisora Patricia Arribas quien nos animó a realizar nuestro primer trabajo de investigación y sobre todo a la Dra. De Sequera por abrirnos paso en este camino, por que sin ella y todo su empeño y esfuerzo no hubiera sido posible llevar a cabo este estudio.

Recibido: 03 noviembre 2016

Revisado: 10 noviembre 2016

Modificado: 05 diciembre 2016

Aceptado: 10 diciembre 2016

\section{Bibliografía}

1. Barth C, Boer W, Garzoni D, Kuenzi T, Ries W, Schaefer R. Characteristics of hypotension-prone haemodialysis patients: is there a critical relative blood volume?. Nephrol Dial Transplant 2003; 18: 1353-1360.

2. Booth J, Pinney J, Davenport A. Do Changes in Relative Blood Volume Monitoring Correlate to hemodialysis-Associated Hypotension?. Nephron Clin Pract 2011; 117: c179-c183.

3. Benaroia $M$, Iliescu EA. Oral intake during hemodialysis: Is there an association with intradialytic hypotension?. Hemodialysis International 2008; 12:62-65.

4. Zoccali C, Mallamaci M, Ciccarelli M, Maggiore Q. Postpandrial alterations in arterial pressure control during hemodiálisis in uremic patients. Clinical Nephrology 1989; 31: 323-326.

5. Gentilcore D, Jones KL, O’Donovan DG, Horowitz M. Postpandrial Hypotension - Novel Insights into Pathophysiology and Therapeutic Implications. Current Vascular Pharmacology 2006; 4, 161-171.

6. Vázquez M, Gruss E, Peláez A, Gago $M^{\circ} \mathrm{C}$, Martinez $\mathrm{S}$, Pérez J. Influencia de la ingesta intradiálisis en la hipotensión. Listado trabajos premiados 2010. Rev.Soc.Esp.Enf.Nefrol 2010; 384-388. 